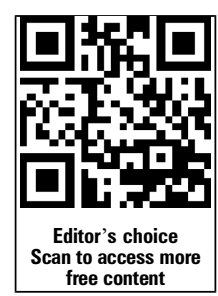

- Additional material is published online only. To view please visit the journal online (http://dx.doi.org/10.1136/ thoraxjnl-2014-206070).

${ }^{1}$ Departments of Clinical Immunology and Allergy, School of Medicine, University of São Paulo, Sao Paulo, Brazil ${ }^{2}$ Department of Physical Therapy, School of Medicine, University of São Paulo, São Paulo, Brazil

${ }^{3}$ Pulmonary Division, Heart Institute (InCor), School of Medicine, University of São Paulo, São Paulo, Brazil ${ }^{4}$ Department of Medicine, Laboratory of Experimental Therapeutics (LIM-20), School of Medicine, University of Sao Paulo, São Paulo, Brazil

\section{Correspondence to} Professor Celso R F Carvalho, Department of Medicine, School of Medicine, University of Sao Paulo, Av. Dr. Arnaldo 455-room 1210, Sao Paulo, SP 01246-903, Brazil; cscarval@usp.br

AF-P and FARM contributed equally to this paper.

Received 22 July 2014 Revised 3 February 2015 Accepted 1 April 2015 Published Online First 10 June 2015

\section{CrossMark}

To cite: França-Pinto $A$, Mendes FAR, de CarvalhoPinto RM, et al. Thorax 2015;70:732-739.

\title{
Aerobic training decreases bronchial hyperresponsiveness and systemic inflammation in patients with moderate or severe asthma: a randomised controlled trial
}

\author{
Andrezza França-Pinto, ${ }^{1}$ Felipe A R Mendes, ${ }^{2}$ Regina Maria de Carvalho-Pinto, ${ }^{3}$ \\ Rosana Câmara Agondi, ${ }^{1}$ Alberto Cukier, ${ }^{3}$ Rafael Stelmach, ${ }^{3}$ \\ Beatriz M Saraiva-Romanholo, ${ }^{4}$ Jorge Kalil, ${ }^{1}$ Milton A Martins, ${ }^{4}$ \\ Pedro Giavina-Bianchi, ${ }^{1}$ Celso R F Carvalho ${ }^{2}$
}

\section{ABSTRACT \\ Background The benefits of aerobic training for the main features of asthma, such as bronchial hyperresponsiveness (BHR) and inflammation, are poorly understood. We investigated the effects of aerobic training on BHR (primary outcome), serum inflammatory cytokines (secondary outcome), clinical control and asthma quality of life (Asthma Quality of Life Questionnaire (AQLQ)) (tertiary outcomes). \\ Methods Fifty-eight patients were randomly assigned} to either the control group (CG) or the aerobic training group (TG). Patients in the CG (educational programme +breathing exercises (sham)) and the TG (same as the CG+aerobic training) were followed for 3 months. BHR, serum cytokine, clinical control, AQLQ, induced sputum and fractional exhaled nitric oxide (FeNO) were evaluated before and after the intervention.

Results After 12 weeks, 43 patients (21 CG/22 TG) completed the study and were analysed. The TG improved in BHR by 1 doubling dose (dd) $(95 \% \mathrm{Cl} 0.3$ to $1.7 \mathrm{dd}$ ), and they experienced reduced interleukin 6 (IL-6) and monocyte chemoattractant protein 1 (MCP-1) and improved AQLQ and asthma exacerbation $(p<0.05)$. No effects were seen for IL-5, IL-8, IL-10, sputum cellularity, FeNO or Asthma Control Questionnaire 7 (ACQ-7; $p>0.05$ ). A within-group difference was found in the ACQ-6 for patients with non-well-controlled asthma and in sputum eosinophil and FeNO in patients in the TG who had worse airway inflammation.

Conclusions Aerobic training reduced BHR and serum proinflammatory cytokines and improved quality of life and asthma exacerbation in patients with moderate or severe asthma. These results suggest that adding exercise as an adjunct therapy to pharmacological treatment could improve the main features of asthma. Trial registration number NCT02033122.

\section{INTRODUCTION}

Asthma, defined as a chronic inflammatory disorder of the airways, is characterised by airway obstruction and bronchial hyperresponsiveness (BHR) and is associated with recurrent episodes of wheezing, breathlessness, chest tightness and coughing. ${ }^{1}$ Asthma symptoms experienced during daily

\section{Key messages}

What is the key question?

- Does aerobic exercise improve bronchial hyperresponsiveness and airway and systemic inflammation in patients with moderate or severe asthma?

What is the bottom line?

- Improvements in aerobic fitness reduced bronchial hyperresponsiveness in one doubling dose of histamine and reduced systemic inflammation in patients with moderate or severe asthma under optimal medical treatment, suggesting that this therapy is an important adjuvant in asthma treatment.

\section{Why read on?}

- This randomised and controlled trial provides the first evidence, obtained using a gold-standard method, that improvement in aerobic exercise reduces bronchial hyperresponsiveness and systemic inflammation.

physical activities or the fear of triggering asthma may keep patients with asthma from engaging in physical exercise, ${ }^{2}$ which often leads to a detrimental health cycle and an aversion to exercise, and reduces activity in daily life and physical fitness. ${ }^{34}$ Interestingly, a low level of physical activity has been strongly and independently associated with increased BHR in patients with asthma.

However, exercise training has been proposed as an adjunctive therapy in asthma treatment because it improves physical fitness, health-related quality of life (HRQoL) ${ }^{6}$ and asthma symptoms, ${ }^{7}$ and because it reduces corticosteroid consumption. ${ }^{8}$ However, the effects of exercise training on BHR remain controversial. Two recent systematic reviews evaluated the effects of aerobic training on BHR and reported either no benefit ${ }^{9}$ or only a trend towards lower BHR after exercise training. ${ }^{10}$ 
The lack of evidence observed in these reviews is explained by the great diversity in patient disease severity, clinical control status and medication management. In addition, BHR in these studies $^{9}{ }^{10}$ was not properly evaluated using a doubling dose, which is the gold-standard method recommended by current guidelines and has been widely used in clinical trials. ${ }^{11}$ As a consequence, both meta-analyses recommended performing well designed trials using standardised tools and more detailed sample characterisation to investigate the potential benefits of regular exercise on BHR in patients with asthma.

Although the effect of aerobic training on BHR in patients with asthma remains poorly understood, studies in asthma animal models have demonstrated that exercise training reduces airway responsiveness and inflammation. ${ }^{12} 13$ Three potential mechanisms have been proposed: reductions in expression of the T helper 2 (Th2) cytokines interleukin (IL)-4, IL-5 and IL-13, ${ }^{13-16}$ reductions in the chemokines monocyte chemoattractant protein $(\mathrm{MCP})^{16}$ and keratinocyte chemoattractant (KC; murine homologue to human IL-8) ${ }^{15}$ and increases in the anti-inflammatory cytokine IL-10. ${ }^{13}{ }^{16}$ To the best of our knowledge, no previous study has investigated such mechanisms in patients with asthma. Given that BHR and inflammation are characteristic features of asthma and given that exercise has systemic anti-inflammatory effects, ${ }^{17}$ the aim of the present study was to investigate the effects of aerobic training on BHR (primary aim) and serum inflammatory cytokines (secondary aim). In addition, clinical control, asthma quality of life and airway inflammation were evaluated (tertiary outcomes).

\section{METHODS}

Detailed study methods are provided in the online supplementary appendix.

\section{Subjects}

Outpatients with moderate or severe persistent asthma, aged between 20 and 59 years, were recruited from a University Hospital. The Ethics Review Board of the Clinical Hospital approved the study (protocol 0121/10). All patients signed an informed consent form and the trial was registered at ClinicalTrials.gov (NCT02033122). Asthma was diagnosed according to the Global Initiative for Asthma, ${ }^{1}$ and disease severity was determined by combining the current level of symptoms, pulmonary function and maintenance treatment. ${ }^{18}$ The patients were managed under optimal medical treatment, monitored by pulmonologists for at least 6 months and considered clinically stable (without exacerbations or changes in medication for at least 30 days).

Patients who met the following criteria were excluded: cardiovascular, musculoskeletal or other chronic lung diseases; current participation in a moderate or vigorous exercise programme; and current smokers or ex-smokers.

\section{Experimental design}

This was a randomised, controlled and single-blinded trial that included an intervention of an aerobic training programme. The study was performed between two medical visits, and during the intervention period, the pharmacotherapy was maintained. Before and after the intervention, BHR, serum levels of cytokines, total immunoglobulin $\mathrm{E}$ (IgE), induced sputum, fractional exhaled nitric oxide (FeNO), clinical control (exacerbation, diary of daily symptoms and Asthma Control Questionnaire (ACQ)), Asthma Quality of Life Questionnaire (AQLQ), pulmonary function and exercise capacity were assessed.
After the baseline evaluation, the eligible patients were randomly assigned following simple randomisation procedures (drawing of a sealed opaque envelope containing group code control group (CG) or training group (TG)) by a researcher not involved in the study. The CG patients were subjected to a breathing exercise programme (sham intervention), and the TG patients were subjected to the same breathing exercise programme and an aerobic exercise training programme. Both groups also underwent a $4 \mathrm{~h}$ educational programme. All patients completed the 24 treatment sessions, after which they were reevaluated.

\section{Interventions}

Breathing exercise programme

Both groups completed a yoga breathing exercise programme twice a week for 12 weeks. ${ }^{67}$ Each session lasted 30 min and was supervised by a physiotherapist. Breathing exercises were included as a sham intervention in the CG to prevent differences in the number of hospital visits and to reduce possible differences in the amount of attention between groups but not to induce benefits in patients with asthma. ${ }^{67}$

\section{Aerobic training programme}

All subjects from the TG completed the aerobic training programme twice a week for 12 weeks on an indoor treadmill. Each aerobic training session lasted $35 \mathrm{~min}$ and was divided into $5 \mathrm{~min}$ of warm-up, $25 \mathrm{~min}$ of aerobic training and $5 \mathrm{~min}$ of cool-down. ${ }^{6}$ At the end of the programme, all the subjects were performing vigorous training, based on the anaerobic threshold $(\mathrm{AnT})$ and the respiratory compensation point.

\section{Assessments}

\section{Bronchial hyperresponsiveness}

A bronchial provocation test with histamine was conducted according to American Thoracic Society (ATS) guidelines. ${ }^{19}$ The test was considered positive when the histamine concentration promoted a decrease $\geq 20 \%$ in forced expiratory volume in $1 \mathrm{~s}$ $\left(\mathrm{FEV}_{1}, \mathrm{PC}_{20}\right)$.

\section{Serum cytokines and total lgE}

The cytometric bead array method (BD Biosciences, San Jose, California, USA) was used to analyse the levels of IL-4, IL-5, IL-6, IL-10, tumour necrosis factor (TNF)- $\alpha$, IL-12p70, IL-8/ CXCL8, MCP-1/CCL2 and RANTES/CCL5. Total serum IgE was measured by nephelometry using commercially available kits (Dade Behring/Siemens, Deerfield, Illinois, USA).

\section{Fractional exhaled nitric oxide}

All measurements were determined by chemiluminescence (Sievers 280) in accordance with the ATS recommendations. ${ }^{20}$ FeNO values were considered elevated at $>26 \mathrm{ppb}^{21}$

\section{Induced sputum}

Sputum was collected and processed using a standard method. ${ }^{22}$ Eosinophil values were considered elevated at $>3 \%{ }^{23}$

\section{Asthma symptoms and exacerbation}

Asthma symptoms and exacerbation were evaluated using a daily diary of symptoms as previously reported. ${ }^{67} \mathrm{~A}$ day was considered free of asthma symptoms when the patient did not report any symptoms, and these days were totalled monthly. Asthma exacerbation was defined as an increase in symptoms associated with at least one of the following criteria: use of rescue medication $\geq 4$ puffs per $24 \mathrm{~h}$ during a $48 \mathrm{~h}$ period, need 
for systemic corticosteroids, unscheduled medical appointment, visit to an emergency room or hospitalisation.

\section{Asthma control questionnaire}

The ACQ-7 consists of seven questions related to asthma symptoms, use of short-acting $\beta_{2}$ agonists and $\mathrm{FEV}_{1}$ in the percent of predicted values. The ACQ-6 is the same as the ACQ-7 without the question related to $\mathrm{FEV}_{1}{ }^{24}$

\section{Asthma quality of life}

Asthma quality of life was assessed using the AQLQ, ${ }^{25}$ which has four domains: activity limitations, symptoms, emotional function and environmental stimuli. A higher AQLQ score indicates a better quality of life. ${ }^{25}$

Cardiopulmonary exercise test and pulmonary function

The test was performed on a treadmill with a ramp protocol, as recommended by the American College of Cardiology/American Heart Association. ${ }^{26}$ Pulmonary function testing was performed according to the current ATS/European Respiratory Society guidelines. $^{27}$
Atopy

Patients were considered atopic if they presented a clinical history suggestive of respiratory allergy and specific $\operatorname{IgE}$ antibodies in the following tests: in vivo (skin prick test) and/or in vitro (Phadiatop test).

\section{Statistical analysis}

A sample size of 34 patients (17 in each group) was estimated to provide $80 \%$ power to detect a 1 doubling-dilution shift in histamine $\mathrm{PC}_{20}$ value (minimal clinical difference), assuming a 1.0 within-patient SD in doubling-dilution shift and an $\alpha$ of 0.05 (two tailed). ${ }^{28} \quad \mathrm{PC}_{20}$ histamine changes were expressed in terms of doubling dose (dd) concentrations, calculated as $\Delta \log \mathrm{PC}_{20}$ $\log 2,{ }^{28}$ and Student's t test was used to compare groups. ACQ, AQLQ, aerobic capacity and pulmonary function were summarised using means and SDs, and differences between the CG and TG were compared using Student's unpaired t test. Sputum cell counting, FeNO, cytokine concentrations and total IgE were summarised using medians and IQRs (25\% and 75\%), and differences between the CG and TG were compared using the unpaired Mann-Whitney U test. The proportion of patients experiencing exacerbations between the TG and placebo CG were compared by

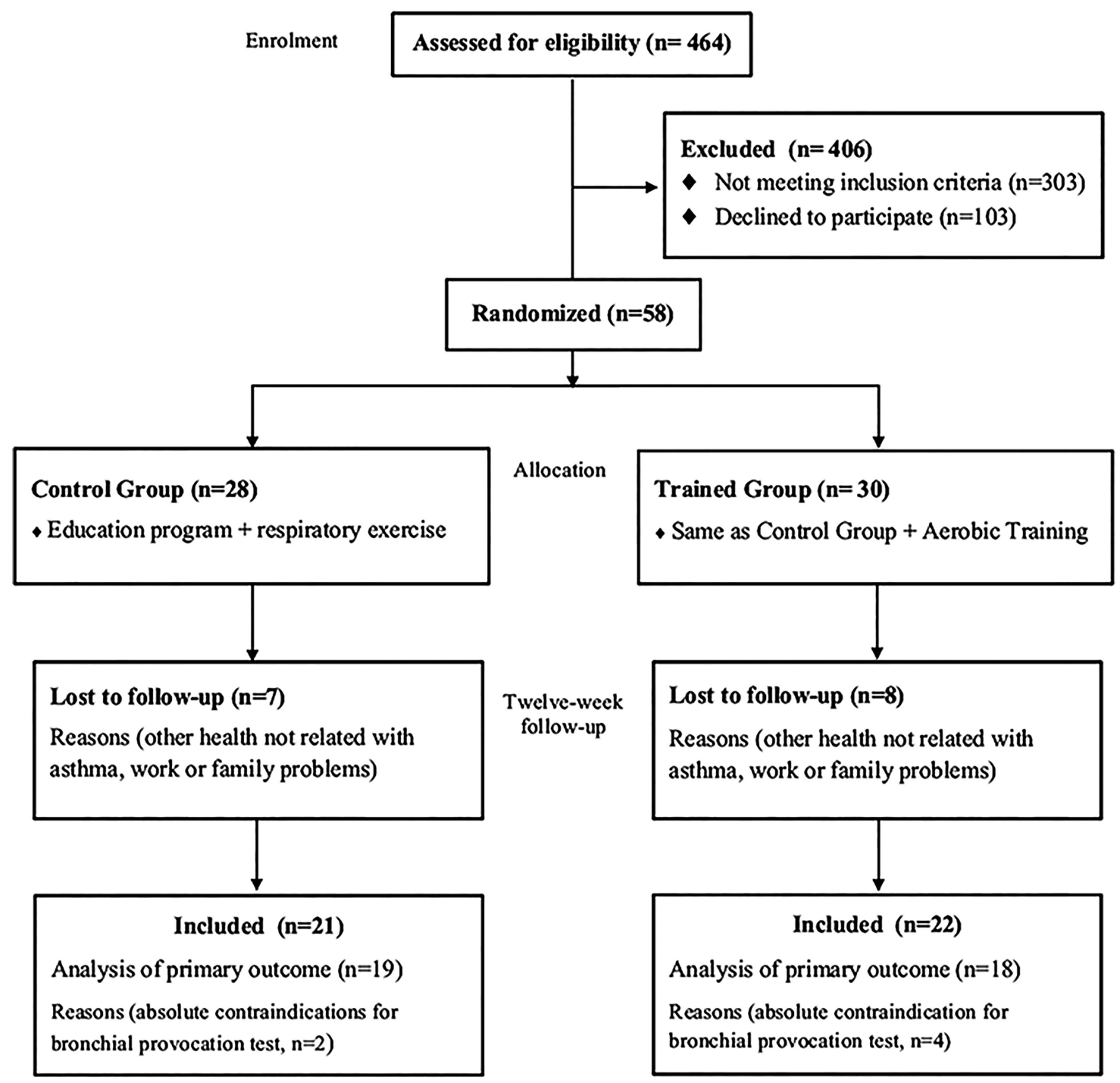

Figure 1 Flow of participants through the study (CONSORT diagram). CG, control group; TG, training group. 
Table 1 Baseline characteristics of patients with asthma

\begin{tabular}{|c|c|c|}
\hline Patient characteristics & $\begin{array}{l}\text { Control group } \\
(n=21)\end{array}$ & $\begin{array}{l}\text { Training group } \\
(n=22)\end{array}$ \\
\hline \multicolumn{3}{|l|}{ Anthropometric data } \\
\hline Sex (F/M) & $17 / 4$ & $17 / 5$ \\
\hline Age, years old; mean (SD) & $44(9)$ & $40(11)$ \\
\hline BMI, kg/m²; mean (SD) & $26.4(4.3)$ & $26.5(4.2)$ \\
\hline \multicolumn{3}{|l|}{ Medication } \\
\hline $\begin{array}{l}\text { Budesonide dosage, } \\
\mu \mathrm{g} / \text { day; mean (SD) }\end{array}$ & $804(370)$ & 909 (594) \\
\hline $\begin{array}{l}\text { Long-acting } \beta_{2} \text { agonists, } \\
\mu \mathrm{g} / \text { day; mean (SD) }\end{array}$ & $34.5(32.1)$ & $26.7(17.7)$ \\
\hline Onset of asthma in childhood, $\mathrm{n}(\%)$ & $12(57)$ & $17(77)$ \\
\hline IgE, IU/mL; median (25th-75th) & $289.0(57-877)$ & $451.5(151-1183)$ \\
\hline Atopy, n (\%) & $15(71.4)$ & $20(91.0)$ \\
\hline $\mathrm{BHR}, \mathrm{PC}_{20}, \mathrm{mg} / \mathrm{mL}$; median (25th-75th) & $0.5(0.3-1.7)$ & $0.3(0.2-0.5)$ \\
\hline Eosinophils, \%; median (25th-75th) & $6.1(9)$ & $10.1(12)$ \\
\hline FeNO, ppb; median (25th-75th) & $26.7(22.5-38.9)$ & $32.0(21.1-44.8)$ \\
\hline ACQ-7, score; mean (SD) & $1.6(0.9)$ & $1.4(1.2)$ \\
\hline $\begin{array}{l}\text { Exacerbations in the last } 12 \text { months; } \\
\text { no. events/patients }\end{array}$ & 1.9 & 1.2 \\
\hline AQLQ, total score; mean (SD) & $4.2(1.1)$ & $4.6(1.4)$ \\
\hline $\begin{array}{l}\text { Aerobic capacity, } \mathrm{VO}_{2 \max }, \mathrm{mL} / \mathrm{kg} / \mathrm{min} \text {; } \\
\text { mean (SD) }\end{array}$ & $25.5(5.9)$ & $27.0(4.3)$ \\
\hline \multicolumn{3}{|l|}{ Pulmonary function } \\
\hline $\mathrm{FEV}_{1}, \%$; mean (SD) & $66.3(19.0)$ & $69.0(21.0)$ \\
\hline $\mathrm{FEV}_{1} / \mathrm{FVC}$, \%; mean (SD) & $72.2(10.0)$ & $73.0(10.5)$ \\
\hline
\end{tabular}

$2 \times 2$ contingency tables using the $\chi^{2}$ test. Within-group differences were compared by the paired test. The level of significance was set at $5 \%(p<0.05)$ for all the tests. The statistical analysis was blinded to the treatment allocation and was performed using statistical software (SigmaStat 3.5, Systat Software Inc).

\section{RESULTS}

A total of 464 subjects were assessed for eligibility: 303 were excluded, 103 refused to participate and 58 patients were randomised into two groups. Forty-three patients completed the study and were analysed (21 CG/22 TG) (figure 1). Both groups had similar baseline characteristics (table 1 ).

\section{Bronchial hyperresponsiveness}

Six patients (2 CG, 4 TG) were not able to perform the bronchial provocation test because they had $\mathrm{FEV}_{1}<1.0 \mathrm{~L}$ after medication was withdrawn for $12 \mathrm{~h}$ during the initial evaluation. At baseline, two patients were classified as borderline, five were classified as mildly hyperresponsive and 29 were classified as moderately to severely hyperresponsive. After the intervention, the BHR decreased in the TG $(\mathrm{n}=18)$, with an increment in $\mathrm{PC}_{20}$ of $1 \mathrm{dd}$ (95\% CI 0.3 to $1.7 \mathrm{dd})$, and did not change in the CG $(n=19)$ (0.06 dd; $95 \%$ CI -0.6 to $0.7 \mathrm{dd})(\mathrm{p}=0.039$; figure 2$)$.

\section{Cytokine and chemokine levels and total IgE}

The CG and TG had similar baseline levels of cytokines (IL-5, IL-6, IL-8 and IL-10), but MCP-1 was higher in the TG $(p=0.002)$ (table 2). There were significant reductions in IL-6 $(p=0.042)$ and MCP-1 $(p=0.045)$ in the TG compared with

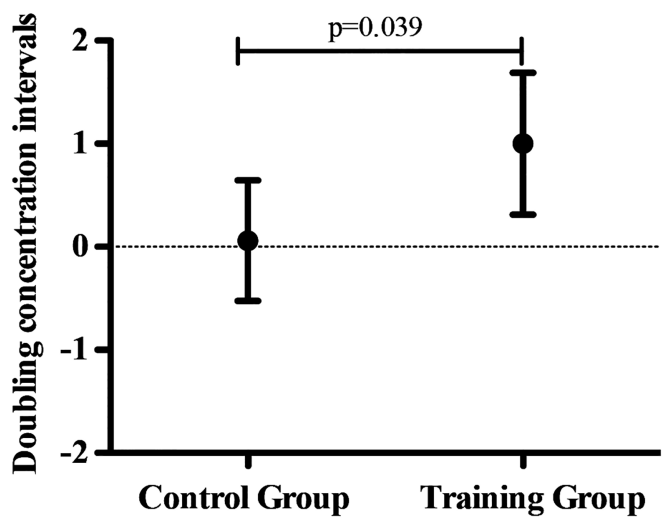

Figure 2 Effect of aerobic training exercise on bronchial hyperresponsiveness in patients with asthma (control group, $C G, n=18$; training group, TG, $\mathrm{n}=18$ ). Data are presented as means and $95 \% \mathrm{Cls}$ of the doubling dose concentration.

the CG (table 2). IL-8 was decreased in the TG, but the differences between groups were not significant $(p=0.055)$. IL-5, IL-10 and IgE did not significantly change $(\mathrm{p}>0.05)$ (table 2$)$. IL-4, TNF- $\alpha$ and RANTES were outside the limit of detection of the assay and could not be analysed.

\section{Clinical asthma control}

The number of days free of asthma symptoms increased in the TG after the intervention $(p=0.042)$, with no difference between the groups $(p=0.987$, table 3$)$. The frequency of exacerbations during treatment was lower in the TG compared with the CG (0.6 vs 1.5 exacerbations/patient; $\mathrm{p}=0.021)$. According to ACQ-7 before intervention, 12 patients were classified as having controlled asthma $(<0.75), 12$ partially controlled $(0.75-1.5)$ and 19 uncontrolled $(>1.5)$. TG patients with non-well-controlled asthma (ACQ-6 $>0.75$ points, $n=14$ ) presented an improvement after aerobic training $(p=0.001)$, with no differences between the groups $(p=0.248$, figure 3D). The same analysis using the ACQ-7 demonstrated no difference within or between the groups $(\mathrm{p}=0.785)$ (figure $3 \mathrm{C})$.

\section{Asthma quality of life questionnaire}

Between-group differences were observed in the activity limitation $(p=0.009)$ domains and in the AQLQ total score $(p=0.034)$ in favour of the TG (table 3). Significant within-group improvement in the emotional function domain was seen in the TG $(p=0.005)$, with no difference between the groups $(p=0.084$, table 3$)$. Fifteen patients $(68 \%)$ from the TG showed a clinically significant improvement in AQLQ total score $(\geq 0.5$ points). The TG presented a linear relationship between improvements in the ACQ-7 and AQLQ $(r=-0.74, p<0.001)$

\section{Induced sputum cellularity and FeNO}

The intervention did not induce a significant change in either sputum cellularity $(p=0.648)$ or $\mathrm{FeNO}$ in either group $(\mathrm{p}=0.397)$ (table 3). Patients from the TG with increased eosinophilic inflammation $(>3 \%, \mathrm{n}=13)$ or FeNO $(>26.0 \mathrm{ppb}$, $\mathrm{n}=12$ ) at baseline presented a significant reduction in these values ( $p=0.015$ and 0.019 , respectively), but the differences between the groups were not significant $(p=0.533$ and 0.452 , respectively; figures $3 \mathrm{~A}, \mathrm{~B}$ ). Eight patients in the CG and 9 in the TG presented increased eosinophilic inflammation and FeNO. The TG presented a linear relationship between baseline eosinophil counts and reduction after exercise training (in delta, 
Table 2 Within-group comparison and between-group comparison for cytokine levels and total IgE in patients with asthma

\begin{tabular}{|c|c|c|c|c|c|c|c|c|}
\hline \multirow[b]{2}{*}{ Outcomes } & \multicolumn{3}{|l|}{ Control group $(n=21)$} & \multicolumn{3}{|l|}{ Training group $(n=22)$} & \multicolumn{2}{|l|}{ Treatment effect } \\
\hline & Before & $\begin{array}{l}\text { Mean }(95 \% \mathrm{Cl}) \\
\text { within-group } \\
\text { difference }\end{array}$ & $\begin{array}{l}p \text { Value } \\
\text { time }\end{array}$ & Before & $\begin{array}{l}\text { Mean }(95 \% \mathrm{Cl}) \\
\text { within-group } \\
\text { difference }\end{array}$ & $\begin{array}{l}\mathrm{p} \text { Value } \\
\text { time }\end{array}$ & $\begin{array}{l}\text { Mean }(95 \% \mathrm{Cl}) \\
\text { e between-group } \\
\text { difference }\end{array}$ & $\begin{array}{l}\mathrm{p} \text { Value } \\
\text { treatment }\end{array}$ \\
\hline IL-5 (fg/mL) & 129.2 (80.1 to 205.3 ) & $46.8(-3.3$ to 95.6$)$ & 0.066 & $155.5(87.4,-170.3)$ & $2.0(-21.2$ to 25.1$)$ & 0.862 & $-19.4(-53.3$ to 14.4$)$ & 0.252 \\
\hline IL-6 (fg/mL) & 298.2 (162.8 to 633.9$)$ & $67.6(-186.7$ to 322.0$)$ & 0.585 & 258.7 (214.5 to 467.6$)$ & 212.6 (83.0 to 341.7 ) & 0.003 & 207.1 (7.7 to 406.1 ) & 0.042 \\
\hline IL-8 (fg/mL) & 1713.9 (1392 to 1858$)$ & $51.7(-185.9$ to 289.6$)$ & 0.655 & 1564.0 (1115 to 1941$)$ & 318.8 (76.0 to 561.6$)$ & 0.013 & $127.3(-5.4$ to 508.6$)$ & 0.055 \\
\hline IL-10 (fg/mL) & $100.7(1.0$ to 166.7$)$ & $21.3(-16.4$ to 58.9$)$ & 0.253 & 95.4 (1.0 to 123.9$)$ & $17.6(-16.3$ to 51.5$)$ & 0.291 & $10.7(-39.1$ to 60.5$)$ & 0.667 \\
\hline MCP-1 (pg/mL) & 14.1 (4.5 to 19.3$)$ & $0.5(-2.8$ to 3.9$)$ & 0.743 & 20.6 (17.1 to 26.7$)$ & $4.5(-0.4$ to 9.0$)$ & 0.052 & $-5.3(-10.5$ to -0.1$)$ & 0.045 \\
\hline $\lg E(I U / m L)$ & 289.0 (60.5 to 878.5$)$ & $65.4(-133.3$ to 264.1$)$ & 0.500 & 360.5 (78.5 to 993.2$)$ & $-238.5(-1066.3$ to 589.4$)$ & 0.555 & $-280.4(-1144$ to 583.7$)$ & 0.516 \\
\hline
\end{tabular}

Values are presented as medians and (25th-75th) percentiles.fg, femtogram; IgE, immunoglobulin E; IL, interleukin; MCP-1, monocyte chemotactic protein 1; pg, picogram.

$\Delta$ final-initial $)(\mathrm{r}=-0.51 ; \mathrm{p}=0.012)$. Similar results were observed in FeNO levels $(\mathrm{r}=-0.61 ; \mathrm{p}=0.008)$.

\section{Maximal aerobic capacity and pulmonary function}

TG patients experienced increased maximal oxygen consumption $\left(\mathrm{VO}_{2} \max \right)(\mathrm{p}=0.019)$ and aerobic power $(\mathrm{p}=0.029)$ compared with CG patients (table 3 and see online supplementary appendix table S1). No changes in spirometry were observed in either group $(p>0.05)$ (table 3 and see online supplementary appendix table S2).

\section{DISCUSSION}

The results of the current study demonstrate that a 12-week aerobic training programme reduces BHR and serum proinflammatory cytokines and improves quality of life and asthma exacerbation in adults with moderate to severe persistent asthma. In addition, it seems that aerobic training reduces sputum eosinophils and $\mathrm{FeNO}$ in patients with higher inflammation and improves clinical control in patients with worse asthma control.

We are aware of only two randomised controlled trials that evaluated the effect of exercise training on BHR in adults with asthma, and their results are controversial. ${ }^{29}{ }^{30}$ Arandelovic et al ${ }^{29}$ found a significant improvement in histamine $\mathrm{PC}_{20}$ after 6 months of swimming training in patients with mild asthma who were treated with low doses of medication and had normal baseline pulmonary function. In contrast, Cochrane and Clark ${ }^{30}$ reported no change in histamine $\mathrm{PC}_{20}$ after 3 months of land aerobic training in patients with mild or moderate asthma using a higher dose of medication and with worse baseline pulmonary function. The discrepancy between these studies may have been multifactorial and depends on patient characteristics (disease severity, atopy, pharmacotherapy), exercise training programme (duration and intensity), and methodology of BHR analysis. The current study introduces several aspects that merit consideration and certainly add information to explain the effect of aerobic training on asthma pathophysiology for several reasons: this is the first study to observe a clinically significant increase of one doubling concentration in BHR, the proper methodology according the guideline; ${ }^{11}$ the benefit to BHR observed in our study may be explained only by the aerobic training because our patients were under proper medical treatment, in accordance with the recommended guidelines; ${ }^{1}$ we have studied patients with moderate to severe asthma, who often have a greater degree of BHR; ${ }^{31}$ and finally, in our study the patients' clinical characteristics were thoroughly assessed, and the training programme was carefully monitored. These data strongly suggest that the observed effect in the BHR was relevant and was a direct result of the aerobic training.

By definition, BHR in asthma is associated with ongoing airway inflammation, and experimental studies in asthma animal models from our group and other groups have systematically shown that exercise reduces airway inflammation and remodelling. ${ }^{13-16}$ These effects seem to occur due to decreases in Th2 cytokines (IL-4, IL-5 and IL-13) ${ }^{13-16}$ and chemokines (MCP-1 and IL-8), ${ }^{13}{ }^{16}$ and increases in the expression of the antiinflammatory cytokine IL-10. ${ }^{13}{ }^{16}$ In the present study, we investigated these mechanisms and observed that aerobic training reduced serum proinflammatory mediators IL-6 and MCP-1; unlike the results in asthma animal models, we did not observe any effect on IL-5, IL-10 and IL-8. Although it is not possible to establish a direct association among the reduction of BHR and IL-6, and MCP-1 observed in our study, there is enough evidence in the literature demonstrating the importance of these cytokines in airway inflammation and BHR in asthma. ${ }^{32}$ Additionally, we observed a within-group reduction in sputum eosinophil and FeNO in patients in the TG with worse airway inflammation, and that improvement was correlated with the baseline values, in agreement with previous findings from our group. This suggests that the benefits of aerobic training were associated with baseline airway inflammation. ${ }^{7}$ Interestingly, a recent study also observed a reduction in serum IL-6 and sputum eosinophils and neutrophils in obese patients with asthma submitted to exercise training and dietary changes. Taken together, these results indicate that exercise may have an anti-inflammatory effect in distinct asthma phenotypes. ${ }^{33}$

We also observed that aerobic training improved clinical control by reducing exacerbations in TG compared with CG. However, the ACQ-7 was not different between groups. Turner et $a l^{34}$ and Dogra et $a l^{35}$ also observed that aerobic training does not modify clinical control as evaluated by the ACQ-7; however, Dogra et al $l^{35}$ observed an improvement in patients with partially controlled asthma using the ACQ-6 (ACQ-7 without the $\mathrm{FEV}_{1}$ question). Similarly, significant within-group improvements in ACQ-6 were found in patients with non-well-controlled asthma from the TG, demonstrating that the improvement in the ACQ with aerobic training seems to be better quantified by using the ACQ-6 rather than the ACQ-7. These results may be explained by the widely known fact that aerobic training does not improve lung function. ${ }^{4}$ We also showed an average improvement in AQLQ score of 0.8 in the TG that is similar to the improvement observed by Turner et $a l^{34}(0.8)$ and Dogra et $a l^{35}(1.0)$, thereby confirming the importance of regular exercise to improve health-related quality of life, even in patients with asthma undergoing clinical treatment.

Certain limitations need to be addressed when interpreting our results. We evaluated the serum cytokine levels, which may not necessarily reflect airway inflammation; however, it has been extensively demonstrated that the effects of exercise training are more pronounced in the systemic immune response. ${ }^{17}$ In addition, the strict inclusion criteria used in our study limit the external validity of our findings; however, this was an important feature of the 
Table 3 Within-group and between-group comparison for induced sputum cellularity, FeNO, clinical control, health-related quality of life, aerobic capacity and pulmonary function of patients with asthma

\begin{tabular}{|c|c|c|c|c|c|c|c|c|}
\hline \multirow[b]{2}{*}{ Outcomes } & \multicolumn{3}{|c|}{ Control group $(n=21)$} & \multicolumn{3}{|l|}{ Training group $(n=22)$} & \multicolumn{2}{|l|}{ Treatment effect } \\
\hline & Before & $\begin{array}{l}\text { Mean }(95 \% \mathrm{Cl}) \\
\text { within-group difference }\end{array}$ & $\mathrm{p}$ Value time & Before & $\begin{array}{l}\text { Mean }(95 \% \mathrm{Cl}) \\
\text { within-group difference }\end{array}$ & $\mathrm{p}$ Value time & $\begin{array}{l}\text { Mean }(95 \% \mathrm{Cl}) \\
\text { between-group difference }\end{array}$ & $\mathrm{p}$ Value treatment \\
\hline \multicolumn{9}{|l|}{ Clinical Control } \\
\hline Asthma symptom-free days & $15.3(11.0)$ & -2.5 (6.2 to 1.2$)$ & 0.180 & $12.0(11.2)$ & $-4.3(-8.4$ to -0.2$)$ & 0.042 & $0.1(-7.2$ to 7.3$)$ & 0.987 \\
\hline ACQ-7 & $1.6(0.9)$ & $0.1(-2.1$ to 0.5$)$ & 0.395 & $1.4(1.2)$ & $0.2(-0.2$ to 0.5$)$ & 0.267 & $0.2(-0.3$ to 0.7$)$ & 0.457 \\
\hline ACQ-6 & $1.5(1.0)$ & $0.1(-2.8$ to 0.6$)$ & 0.502 & $1.2(1.2)$ & $0.2(-0.2$ to -0.6$)$ & 0.236 & 0.3 (0.3 to 0.8$)$ & 0.327 \\
\hline \multicolumn{9}{|l|}{ AQLQ } \\
\hline Overall & $4.2(1.1)$ & $-0.3(-0.8$ to 0.2$)$ & 0.259 & $4.6(1.4)$ & $-0.7(-1.9$ to 0.2$)$ & 0.005 & $-0.9(-1.7$ to -0.1$)$ & 0.034 \\
\hline Activity limitation domain & $3.8(0.9)$ & $-0.2(-0.8$ to 0.4$)$ & 0.433 & $4.3(1.3)$ & $-0.8(-1.2$ to -0.3$)$ & 0.002 & $-1.1(-1.8$ to -0.3$)$ & 0.009 \\
\hline Symptoms domain & $4.8(1.5)$ & $-0.2(-0.9$ to 0.4$)$ & 0.469 & $5.1(1.5)$ & $-0.6(-1.1$ to 0.0$)$ & 0.053 & $-0.7(-1.5$ to 0.1$)$ & 0.091 \\
\hline Emotional function domain & $4.1(1.9)$ & $-0.6(-1.6$ to 0.4$)$ & 0.250 & $4.6(1.8)$ & $-1.0(-1.6$ to -0.3$)$ & 0.005 & $-0.9(-2.0$ to 0.1$)$ & 0.084 \\
\hline Environmental stimuli domain & $3.7(1.8)$ & $-0.5(-1.5$ to 0.6$)$ & 0.359 & $4.5(2.0)$ & $-0.6(-1.3$ to 0.2$)$ & 0.118 & $-0.9(-2.0$ to 0.3$)$ & 0.140 \\
\hline \multicolumn{9}{|l|}{ Induced sputum } \\
\hline Total cell $\left(10^{6} / \mathrm{mL}\right)$ median $(25 \mathrm{th}-75 \mathrm{th})$ & $0.9(0.1-1.4)$ & $-0.8(-1.5$ to 0.2$)$ & 0.055 & $0.8(0.4$ to 1.6$)$ & $0.2(-0.44$ to 0.77$)$ & 0.583 & $0.6(-0.6$ to 1.7$)$ & 0.333 \\
\hline Eosinophils (\%) median (25th-75th) & $6.1(0.25-14.9)$ & $-7.9(-17.7$ to 1.8$)$ & 0.106 & 10.1 (1.6 to 21.9$)$ & $-0.6(-8.8$ to 7.6$)$ & 0.881 & $-8.8(-2.0$ to 0.3$)$ & 0.648 \\
\hline Neutrophils (\%) median (25th-75th) & $33.8(22.1-66.2)$ & $3.4(-6.9$ to 13.7$)$ & 0.500 & $37.4(16.7$ to 57.5$)$ & $1.6(-12.6$ to 15.7$)$ & 0.821 & $1.7(-13.1$ to 16.6$)$ & 0.816 \\
\hline Lymphocytes (\%) median (25th-75th) & $0.0(0.0-0.1)$ & $0.9(-2.7$ to 4.4$)$ & 0.620 & $0.0(0.0$ to 0.8$)$ & $-1.2(-2.9$ to 0.4$)$ & 0.137 & $-1.0(-2.8$ to 0.7$)$ & 0.251 \\
\hline Macrophages (\%) median (25th-75th) & $40.5(11.1-73.1)$ & $1.4(-9.8$ to 12.5$)$ & 0.799 & 43.4 (25.7 to 65.2$)$ & $-0.9(-16.1$ to 14.4$)$ & 0.907 & $-6.7(-22.7$ to 9.2$)$ & 0.248 \\
\hline FeNO (ppb) median (25th-75th) & $26.7(22.5-38.9)$ & $-5.9(-5,8$ to 4.6$)$ & 0.815 & 32.0 (21.1 to 44.8$)$ & $4.5(-0.7$ to 9.7$)$ & 0.087 & $4.4(-5.9$ to 14.7$)$ & 0.397 \\
\hline \multicolumn{9}{|l|}{ Exercise capacity } \\
\hline Aerobic capacity $\left(\mathrm{VO}_{2 \max } \mathrm{mL} / \mathrm{kg} / \mathrm{min}\right)$ & $25.5(5.9)$ & $2.4(-0.2$ to 4.5$)$ & 0.053 & $27.0(4.2)$ & $-1.0(-2.4$ to 0.5$)$ & 0.182 & $-4.8(-8.9$ to -0.8$)$ & 0.019 \\
\hline Maximal workload (watts) & $202.8(67.3)$ & $-3.3(-25.4$ to 18.9$)$ & 0.762 & $190.3(32.3)$ & $-57.1(-73.1$ to -41.1$)$ & $<0.001$ & $-44.1(-83.4$ to -4.8$)$ & 0.029 \\
\hline \multicolumn{9}{|l|}{ Pulmonary function } \\
\hline $\mathrm{FEV}_{1},(\mathrm{~L})$ & $2.00(0.7)$ & $-0.1(-0.2$ to 0.1$)$ & 0.471 & $2.1(0.76)$ & $0.00(-0.1$ to 0.1$)$ & 0.952 & $-0.0(-0.5$ to 0.4$)$ & 0.930 \\
\hline $\mathrm{FEV}_{1} \%$ predicted & $66.3(19.0)$ & $-2.3(-8.6$ to 3.9$)$ & 0.447 & $69.0(21.0)$ & $-1.1(-4.8$ to 2.6$)$ & 0.546 & $2.5(-11.5$ to 16.5$)$ & 0.721 \\
\hline
\end{tabular}

Data are means (SDs) unless otherwise stated.

ACQ, Asthma Control Questionnaire; AQLQ, Asthma Quality of Life Questionnaire; FeNO, fractional exhaled nitric oxide. 

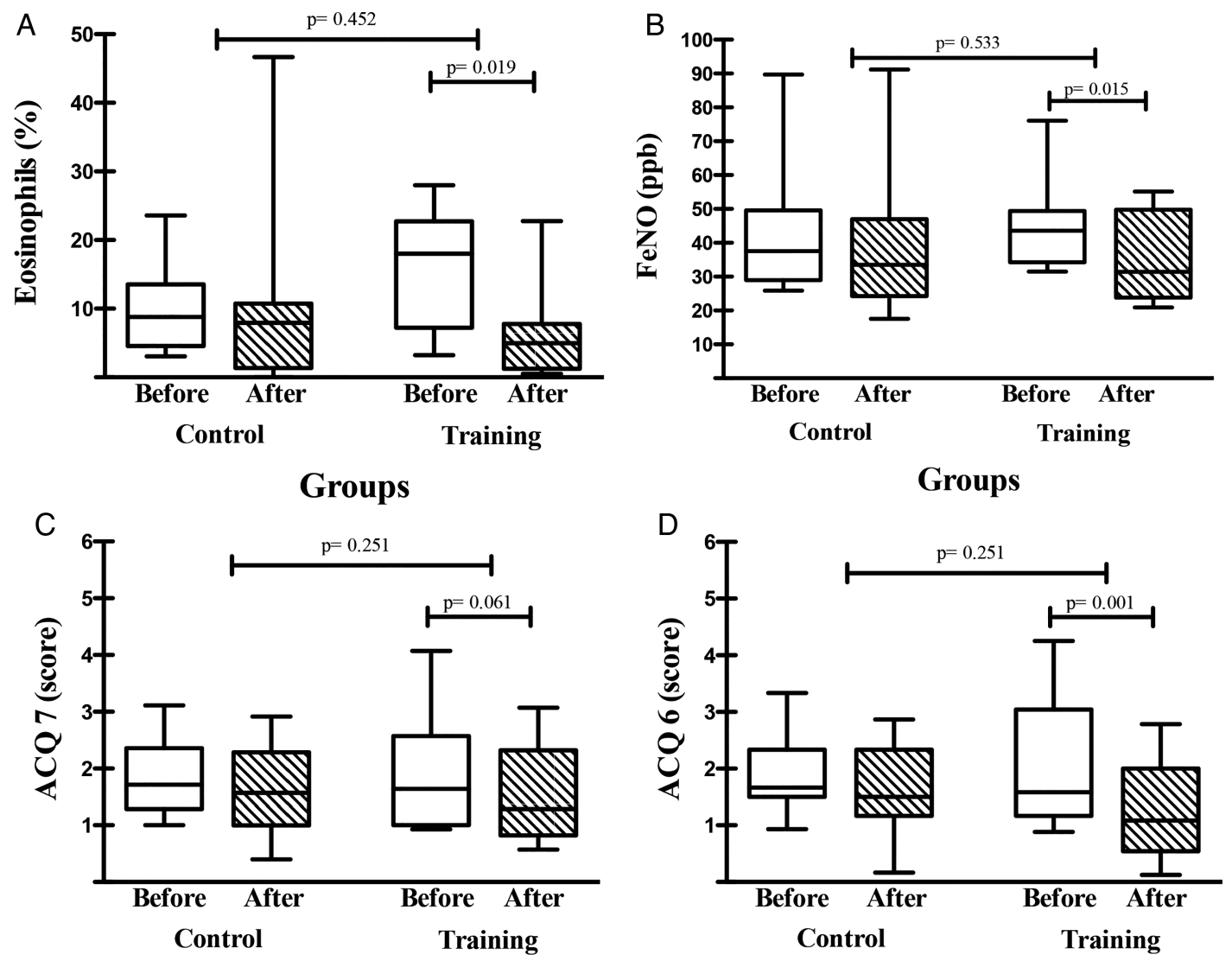

Groups

Groups

Figure 3 Subgroup analysis: (A) Percentage of eosinophils in induced sputum $>3 \%$ (control group, $C G, n=9 ;$ training group, TG, $n=13$ ). (B) Fractional exhaled nitric oxide $>26 \mathrm{ppb}(C G, n=12 ; T G, n=12)$. (C) ACQ-7 >0.75 (CG, $n=17 ; T G, n=14)$. (D) ACQ-6 >0.75 (CG, $n=14 ; T G, n=12)$. ACQ, Asthma Control Questionnaire. Boxes represent the 25th to 75th percentiles, the lines inside the boxes represent the median values, and the bars represent the 10th and 90th percentiles.

study design to reduce variability within the sample for the main outcome (BHR). Finally, for subgroup analysis, significant between-group differences following treatment could not be demonstrated, probably due to the reduced number of individuals, because the sample size was not primarily to evaluate these secondary outcomes. Although, it well known that the key outcome in a clinical trial is the difference between the intervention and CGs, we consider that this within-group difference in the TG was clinically relevant for identifying patients who respond to physical training. As a consequence, this information should subsidise future studies aiming to evaluate differences between treatments to determine the impact of exercise on clinical control and airway inflammation.

In conclusion, our results demonstrate that aerobic training reduces BHR, systemic inflammation and exacerbations and improved quality of life in adults with moderate to severe persistent asthma. In addition, we showed that patients with higher inflammation and lower asthma control obtained greater benefits. These findings suggest that adding exercise as an adjunct therapy to pharmacotherapy can improve the main features of asthma pathophysiology.

Acknowledgements The authors would like to acknowledge: Dr Júlia M D Greve and the exercise physiologist Paulo R Santos-Silva (Institute of Orthopedics and Traumatology, Clinics Hospital, School of Medicine, University of Sao Paulo, São Paulo, Brazil) for their support and blinded analysis of the cardiopulmonary exercise testing; Professor Maria N Sato and PhD student Luanda M S Oliveira (Laboratory of Dermatology and Immunodeficiencies, Department of Dermatology, Medical School, University of São Paulo, São Paulo, Brazil) for conducting the blood sample analysis; and Professor Clarice Tanaka (Department of Physical Therapy, School of Medicine,
University of Sao Paulo, Sao Paulo, Brazil) for her technical support. The authors would also like to acknowledge the American Thoracic Society (ATS) and the Methods in Epidemiologic, Clinical and Operations Research (MECOR) Program for their assistance in the development of research skills in Latin America.

Contributors AF-P: study concept and design, recruitment of patients, manuscript writing and review, data acquisition and results interpretation; FARM (principal contributor guarantor): study concept and design, recruitment of patients, manuscript writing and review; data acquisition; results interpretation and statistical analysis; RMdC-P: patient's medical treatment and manuscript review; RCA: bronchial provocation test support, blinded outcome assessment and manuscript review; $A C$ and RS: study concept and design, results interpretation and manuscript review; BMS-R: blinded outcome assessment, cytospin reading, FeNO analysis, and manuscript review; JK: project supervision and manuscript review; MAM: project supervision, study concept and design and manuscript review; PG-B: project supervision, study concept and design, manuscript review and results interpretation; CRFC: study concept and design, project supervision, results interpretation, overall study coordination, and manuscript writing and review. All the authors have read and approved the final version of the manuscript.

Funding The study was supported by the São Paulo Research Foundation (FAPESP; Grants 2009/53904-9 and 2009/53817-9) and Ministério da Ciência, Tecnologia e Inovação-Conselho Nacional de Pesquisa (CNPq Grants 305987/2010-0).

Competing interests None declared.

Patient consent Obtained.

Ethics approval Clinical Hospital (protocol 0121/10).

Provenance and peer review Not commissioned; externally peer reviewed.

\section{REFERENCES}

1 Global Initiative for Asthma (GINA). Global strategy for asthma management and prevention. Bethesda, MD: National Institutes of Health/National Heart, Lung and Blood Institute of Health, 2012. 
2 Disabella V, Sherman C. Exercise for asthma patients: little risk, big rewards. Phys Sports Med 1998:26:75-85.

3 Villa F, Castro AP, Pastorino AC, et al. Aerobic capacity and skeletal muscle function in children with asthma. Arch Dis Child 2011:96:1-11.

4 Carson KV, Chandratilleke MG, Picot J, et al. Physical training for asthma. Cochrane Database Syst Rev 2013;9:CD001116.

5 Brasholt M, Baty F, Bisgaard H. Physical activity in young children is reduced with increasing bronchial responsiveness. J Allergy Clin Immunol 2010;125:1007-12.

6 Mendes FA, Gonçalves RC, Nunes MP, et al. Effects of aerobic training on psychosocial morbidity and symptoms in patients with asthma: a randomized clinical trial. Chest 2010;138:331-7.

7 Mendes FAR, Mendes FA, Almeida FM, et al. Effects of aerobic training on airway inflammation in asthmatic patients. Med Sci Sports Exerc 2011;43:197-203.

8 Fanelli A, Cabral ALB, Neder JA, et al. Exercise training on disease control and quality of life in asthmatic children. Med Sci Sports Exerc 2007:39:1474-80.

9 Pakhale $S$, Luks V, Burkett $A$, et al. Effect of physical training on airway inflammation in bronchial asthma: a systematic review. BMC Pulm Med 2013;13:38.

10 Eichenberger PA, Diener SN, Kofmehl R, et al. Effects of exercise training on airway hyperreactivity in asthma: a systematic review and meta-analysis. Sports Med 2013;43:1157-70.

11 Reddel HK, Taylor DR, Bateman ED, et al. An official American Thoracic Society/ European Respiratory Society statement: asthma control and exacerbations: standardizing endpoints for clinical asthma trials and clinical practice. Am J Respir Crit Care Med 2009:180:59-99.

12 Hewitt M, Estell K, Davis IC, et al. Repeated bouts of moderate-intensity aerobic exercise reduce airway reactivity in a murine asthma model. Am J Respir Cell Mol Biol 2010;42:243-9.

13 Silva RA, Vieira RP, Duarte ACS, et al. Aerobic training reverses airway inflammation and remodelling in an asthma murine model. Eur Respir J 2010;35:994-1002.

14 Olivo CR, Vieira RP, Arantes-Costa FM, et al. Effects of aerobic exercise on chronic allergic airway inflammation and remodelling in guinea pigs. Respir Physiol Neurobiol 2012;182:81-7

15 Pastva A, Estell K, Schoeb TR, et al. Aerobic exercise attenuates airway inflammatory responses in a mouse model of atopic asthma. $J$ Immunol 2004:172:4520-6.

16 Vieira RP, Claudino RC, Duarte ACS, et al. Aerobic exercise decreases chronic allergic lung inflammation and airway remodelling in mice. Am J Respir Crit Care Med 2007;176:871-7.

17 Gleeson M, Bishop NC, Stensel DJ, et al. The anti-inflammatory effects of exercise: mechanisms and implications for the prevention and treatment of disease. Nat Rev Immunol 2011;11:607-15.

18 Global Initiative for Asthma (GINA). Global strategy for asthma management and prevention. Bethesda, MD: National Institutes of Health/National Heart, Lung and Blood Institute of Health, 2005.
19 Crapo RO, Casaburi R, Coates AL, et al. Guidelines for methacholine and exercise challenge testing-1999. Am J Respir Crit Care Med 2000;161:309-29.

20 American Thoracic Society, European Respiratory Society. Recommendations for standardized procedures for the online and offline measurement of exhaled lowe respiratory nitric oxide and nasal nitric oxide, 2005. Am J Respir Crit Care Med 2005:171:912-30.

21 Shaw DE, Berry MA, Thomas $M$, et al. The use of exhaled nitric oxide to guide asthma management: a randomized controlled trial. Am J Respir Crit Care Med 2007:176:231-7

22 Saraiva-Romanholo BM, Barnabé V, Carvalho AL, et al. Comparison of three methods for differential cell count in induced sputum. Chest 2003;124:1060-6.

23 Green RH, Brightling CE, McKenna S, et al. Asthma exacerbations and sputum eosinophil counts: a randomized controlled trial. Lancet 2002:360:1715-21.

24 Juniper $\mathrm{EF}$, Bousquet J, Abetz L, et al. Identifying 'well-controlled' and 'not well-controlled' asthma using the Asthma Control Questionnaire. Respir Med 2006;100:616-21

25 Juniper EF, Guyatt GH, Epstein RS, et al. Evaluation of impairment of health related quality of life in asthma: development of a questionnaire for use in clinical trials. Thorax 1992:47:76-83.

26 Gibbons RJ, Balady GJ, Bricker JT, et al. ACC/AHA 2002 guideline update for exercise testing: summary article. A report of the American College of Cardiology/ American Heart Association Task Force on Practice Guidelines (Committee to Update the 1997 Exercise Testing Guidelines). J Am Coll Cardiol 2002;40:1531-40.

27 Miller MR, Hankinson J, Brusasco V, et al. Standardization of spirometry. Eur Respir J 2005;26:319-38.

28 Inman MD, Hamilton Al, Kerstjens HAM, et al. The utility of methacholine airway responsiveness measurements in evaluating anti-asthma drugs. J Allergy Clin Immunol 1998;101:342-8.

29 Arandelovic M, Stankovic I, Nikolic M. Swimming and persons with mild persistent asthma. ScientificWorld Journal 2007:7:1182-8.

30 Cochrane LM, Clark CJ. Benefits and problems of a physical training program for asthmatic patients. Thorax 1990;45:345-51.

31 O'Byrne PM, Gauvreau GM, Brannan JD. Provoked models of asthma: what have we learnt? Clin Exp Allergy 2009;39:181-92.

32 Barnes PJ. The cytokine network in asthma and chronic obstructive pulmonary disease. J Clin Invest 2008;118:3546-56.

33 Scott HA, Gibson PG, Garg ML, et al. Dietary restriction and exercise improve airway inflammation and clinical outcomes in overweight and obese asthma: a randomized trial. Clin Exp Allergy 2013;43:36-49.

34 Turner S, Eastwood P, Cook A, et al. Improvements in symptoms and quality of life following exercise training in older adults with moderate/severe persistent asthma. Respiration 2011:81:302-10.

35 Dogra S, Kuk JL, Baker J, et al. Exercise is associated with improved asthma control in adults. Eur Respir J 2011;7:318-23. 